\title{
sience \& A Nondestructive Method to Estimate Plant Height, Stem Diameter and Biomass of Rice under Field Conditions Using Digital Image Analysis
}

\author{
Graduate School of
}

M. Mano* and M. Igawa

*Correspondence author: mano@ chiba-u.jp

\begin{abstract}
Plant phenotyping intends measuring complex plant traits, and is important in agricultural research for enhancing yield improvement. Manual plant phenotyping is laborious and destructive, and hence a less-laborious and non-destructive method is required. Here, we proposed a nondestructive method to estimate continuous data of plant traits such as height, stem diameter and biomass using a low cost time-lapse camera. The camera was installed at a rice field in Japan, and captured images for four target plants every three hour. The plant height and stem diameter were determined from the images by referencing scale bars that were placed next to the target plants and above the ground surface. Both the height and the diameter were compared to directly measured ones, and the relationships between those were in good agreement. Plant volumes were estimated from the height and stem diameter assuming a shape of rice plant is cylindrical. Above ground biomass without panicles was determined by rice plants sampled from the field. The determined biomass increased in proportion to the plant volume, and its relationship used to produce continuous data of the rice biomass. The results suggest that the proposed method can be considered as a useful tool of the plant phenotyping.
\end{abstract}

Key words: Crop biomass, Plant phenotyping and Plant traits and yield

\section{Introduction}

Plant phenotyping is defined as the application of methodologies to measure plant traits such as growth, development, yield, architecture, tolerance ranging from the cellar to the whole plant canopy level (Furbank and Tester, 2011; Dhondt, 2013). Therefore, in agricultural research, field scale experiments in plant phenotyping are an important factor to exploit the high production of food to meet future demand (Furbank and Tester, 2011; McCouch et al., 2013). Until now, plant phenotyping applications for indoor field such as plant factory and greenhouse have been developed using current technologies of sensor devices and image analysis (Hartmann et al., 2011; Constantino et al., 2015). On the other hand, plant phenotyping for outdoor field, which is laborious, time-consuming and often destructive when manually methods are used, applications are still under development (Araus and Cairns, 2014; Friedli et al., 2016; Schima et al., 2016), and less-laborious and -time-consuming, and non-destructive phenotyping methods are required and need to be developed ( $\mathrm{Li}$ et al., 2014; Minervini et al., 2015).

Among crops, rice is the staple food of most Asian countries, such as Bangladesh, India, China and Japan, and several methodologies of plant phenotyping have been proposed. Constantino et al. (2015) developed an image processing system that automates the measuring of height and the counting of tillers of a rice in screenhouses. Jiang et al. (2015) reported that a nondestructive method for estimating the total green leaf area of individual rice using multi-angle color images that were captured at indoors. Sritarapipat et al. (2014) and Tilly et al. (2014) measured rice plant heights at outdoors by using of a digital camera and a terrestrial laser scanner, respectively, and Tilley et al. (2014) estimated above ground biomass from a regression model based on the relationship between the rice plant height and biomass. Yang et al. (2013) discussed the key plant phenotyping technologies and introduced their current application in rice, wheat and barley. Though the several methods have been proposed, those have some limitations, such as the only applicable indoor space, the need of high cost for the instrument and the limited range and/or parameters to be measured. Therefore, in this study, we aim to develop a nondestructive method to estimate continuous plant traits data (i.e. heights, stem diameters and above ground biomass) at a rice field by using a low cost time-lapse camera.

\section{Materials and Methods}

\section{Experimental site}

The experiment was conducted at a rice field located in Ibaraki prefecture, Japan $\left(36^{\circ} 03^{\prime} \mathrm{N}, 140^{\circ} 01^{\prime} \mathrm{E}, 11.2 \mathrm{~m}\right.$ a.s. 1.) during May to September 2014. A rectangular plot of $100 \times 54 \mathrm{~m}$ was cultivated with 'Koshihikari' rice (Oryza sativa L.) using established methods, and the transplanting and harvesting date were May 2nd and September 11th, respectively. Further details of the site and experimental crop are given in previous studies (Miyata et al., 2005; Saito et al., 2005; Mano et al., 2007; Ono et al., 2008, 2013, 2015; Mano, 2017).

\section{Measurement system}

The measurement system is similar to the one used to measure the rice plant heights at the same site (Mano, 2017). A weather proof time-lapse camera with a photo flash attachment (BirdCamPro,WingScapes, Alabaster, AL, USA) was used to capture images of rice, and installed to the height of $61 \mathrm{~cm}$. The distance between the camera and the target rice plants was $290 \mathrm{~cm}$, and in this configuration four rice plants were fully captured in each image. A white wire net was placed just behind the target rice plants to distinguish those from other rice plants in the background. As a reference to determine the plant heights and stem diameter, two $150 \mathrm{~cm}$-long scale bars with $0.5 \mathrm{~cm}$ interval marking were used; one was erected vertically next to the target rice plants and the other one was placed horizontally above the ground surface behind 


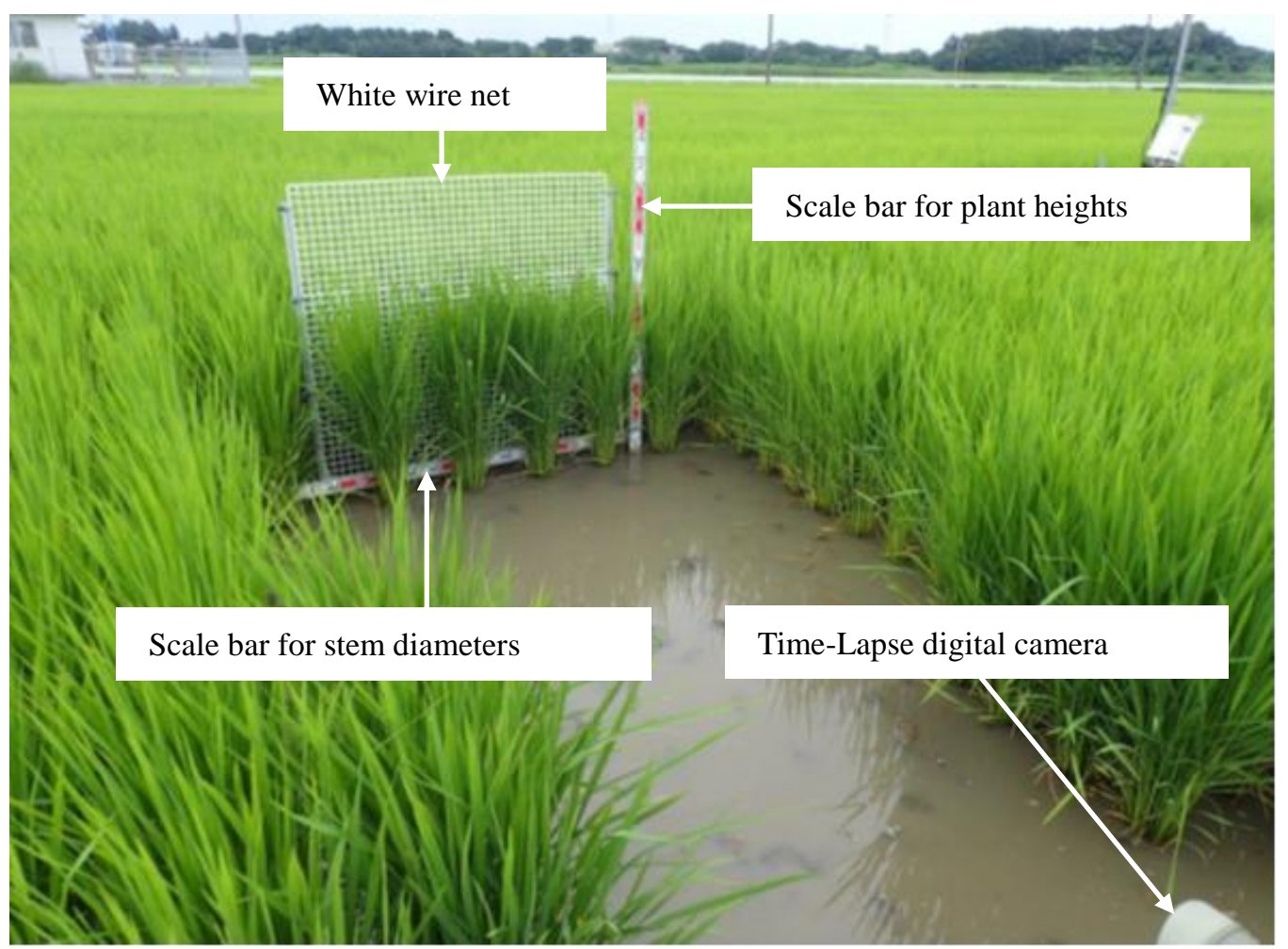

Fig. 1. Photo of the measurement system to estimate the rice plant height and stem diameter

\section{Determine plant heights, stem diameters and biomass}

The camera was set to take images in a three-hour interval during the whole day (an example photo is shown in Fig. 2). Plant heights and stem diameters were determined to $1 \mathrm{~cm}$ resolution from the images by observing the nearest marking on the scale bars where, for the heights, an imaginary horizontal line from the highest point of the target rice plant was crossed, and for the diameters, a length that the target rice plant obstructed. Above ground biomass (hereafter referred to as biomass) was estimated from a regression model based on a similar concept to the pipe model theory (Shinozaki et al., 1964ab) that assumes the relationship between the biomass and plant volumes is linearly proportional. The rice plant volumes were determined from the rice plant
Fig. 1.
-7361 


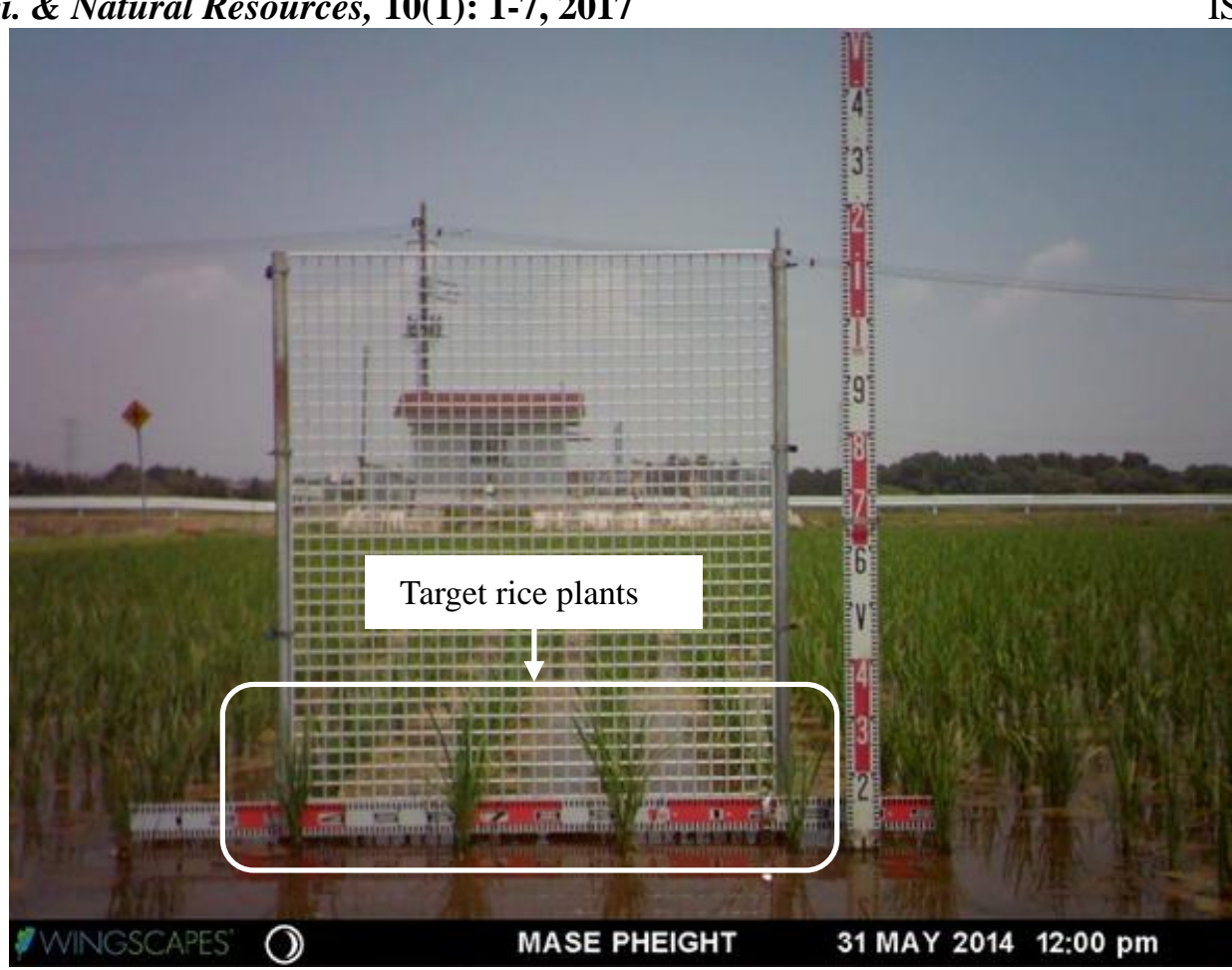

Fig. 2. An example photo taken by the time-lapse camera (BirdCamPro, WingScapes) at 12:00 p.m. on 31st May 2014.

\section{Results and Discussions}

\section{Rice plant height}

For each the target rice plants, the daily average rice plant height was calculated from the plant heights that were determined from the images. Then, the daily average and its standard deviation of the four target rice plant heights were evaluated, and those are shown in Fig. 3(A). The rice plant height increased continuously from ca. $10 \mathrm{~cm}$ at the transplanting in May to a maximum of ca. $130 \mathrm{~cm}$ in early August, and then dropped to ca. $115 \mathrm{~cm}$ may due to lodging by winds, and slightly decreased to ca. $110 \mathrm{~cm}$ until the harvesting. The reference plant heights that were directly measured by the measuring tape, which were averaged from four samples (i.e. four target rice plants)

(A)

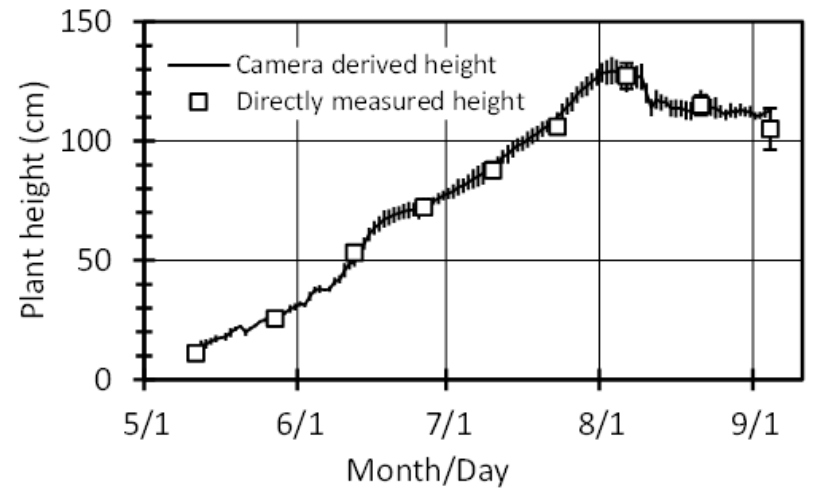

on the sampling day, followed the same seasonal trends, as also shown in Fig. 3(A). Both the camera derived and the directly measured rice plant heights matched well, and those relationship was confirmed by a scatter plot in Fig. 3(B). The linear regression forced through the origin resulted in a slope of 1.02 and an R-squared coefficient $\left(\mathrm{R}^{2}\right)$ of 0.99 (Table 1). To evaluate error statistics, the root mean square error (RMSE) was calculated and it exhibits a small value of $3.6 \mathrm{~cm}$ (Table 1). From the camera derived plant height data, detailed characteristics of the seasonal course of the rice plant height, such as the occurrence date of the maximum plant height and sudden drop of the plant height after its maximum, were recognized.

(B)

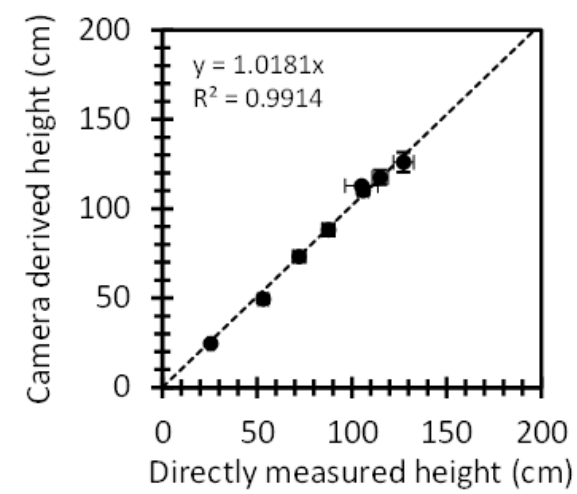

Fig. 3. (A) Seasonal course of the daily average of the rice plant height determined from the camera images (solid line) and directly measured by the measuring tape (square). The standard deviation is shown as error bars. (B) Relationship between the directly measured and the camera derived rice plant heights (error bars are standard deviation). The result of the linear regression model through the origin is presented as a dashed line. 


\section{Rice stem diameter}

The daily average and its standard deviation of the rice stem diameter determined from the images and those determined from directly measured by the measuring tape

(A)

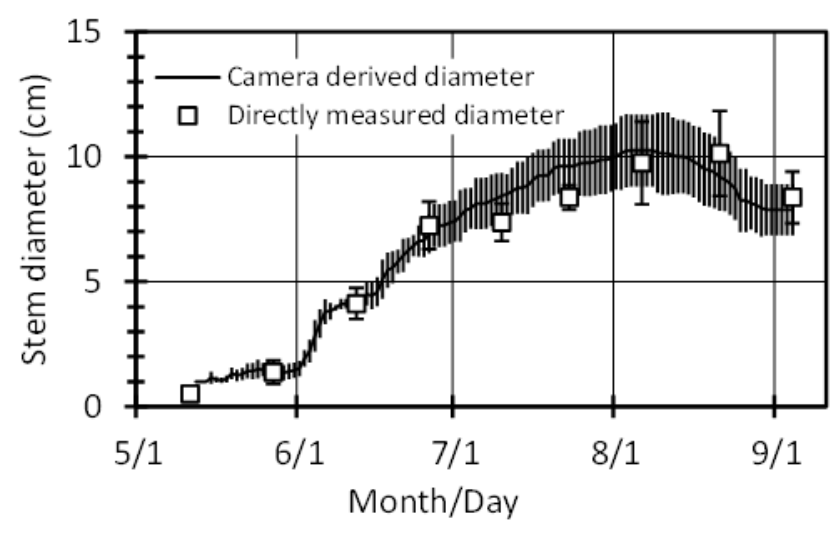

ISSN 1999-7361

were calculated the same way of those plant heights. The camera derived rice stem diameter was ca. 1 to $2 \mathrm{~cm}$ during May, and sharply increased to ca. $4 \mathrm{~cm}$ in early June (Fig. 4(A)).

(B)

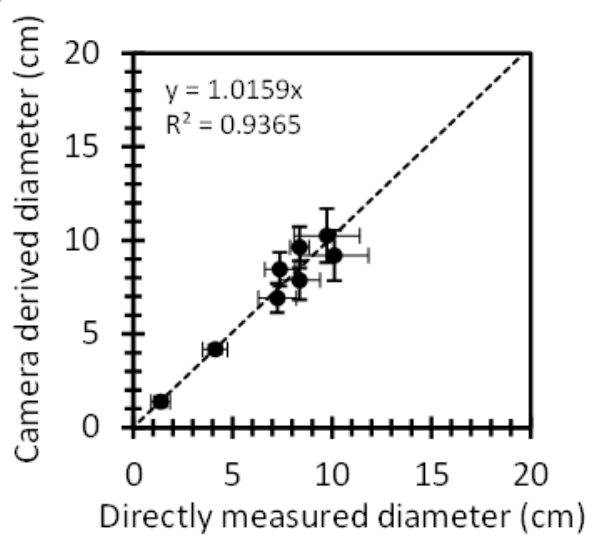

Fig. 4. (A) Seasonal course of the daily average of the rice stem diameter determined from the camera images (solid line) and directly measured by the measuring tape (square). The standard deviations shown as error bars. (B) Relationship between the directly measured and the camera derived rice stem diameters (error bars are standard deviation). The result of the linear regression model through the origin is presented as a dashed line.

Then, it increased continuously to a maximum of ca. 10 $\mathrm{cm}$ in early August and decreased gradually to ca. $8 \mathrm{~cm}$ till the harvesting. To compare with the rice plant height, the ratio of standard deviation to average was larger; it indicated that the difference between each the four rice stem diameter was comparatively large. Though, the relationship between the camera derived and the directly measured rice stem diameters based on daily values was linearly related (Fig. 4[B]), and the parameters of linear regression and error statistics showed a slope of $1.02, \mathrm{R}^{2}$ of 0.94 and RMSE of $0.9 \mathrm{~cm}$ (Table 1). These results lead us to conclude that the camera derived rice plant height and its stem diameter represent the directly measured ones well, where almost no difference (within one standard deviation) with the small RMSE of several centimeters.

Table 1. Results of linear regression analysis between the camera derived plant height/stem diameter (y-axis) and the directly measured ones (x-axis) (column names are "Plant height" and "Stem diameter), and the rice plant volume (x-axis) and the above ground biomass without panicles (y-axis) (column name is "Volume-Biomass"). The linear regression through the origin model was used. For the columns "Plant height" and "Stem diameter", RMSEs are also calculated. The RMSEs are presented in centimeters, and the others are unitless.

\begin{tabular}{cccc}
\hline & Plant height & Stem diameter & Volume-Biomass \\
\hline Slope & 1.02 & 1.02 & 0.0065 \\
$\mathrm{R}^{2}$ & 0.99 & 0.94 & 0.93 \\
RMSE & 3.6 & 0.9 & - \\
\hline
\end{tabular}

\section{Rice biomass}

The volume of rice plant, which shape is assumed as the cylindrical, for each the target rice plant was calculated from the rice plant height and the rice stem diameter determined from the camera images. Then, the average volume of the four target rice plants and its standard deviation on a daily basis were determined. Fig. 5 shows the relationship between the rice plant volume and the dry weight of the rice biomass that were averaged from the ten rice plants sampled from the field at approximately 2-week intervals. 


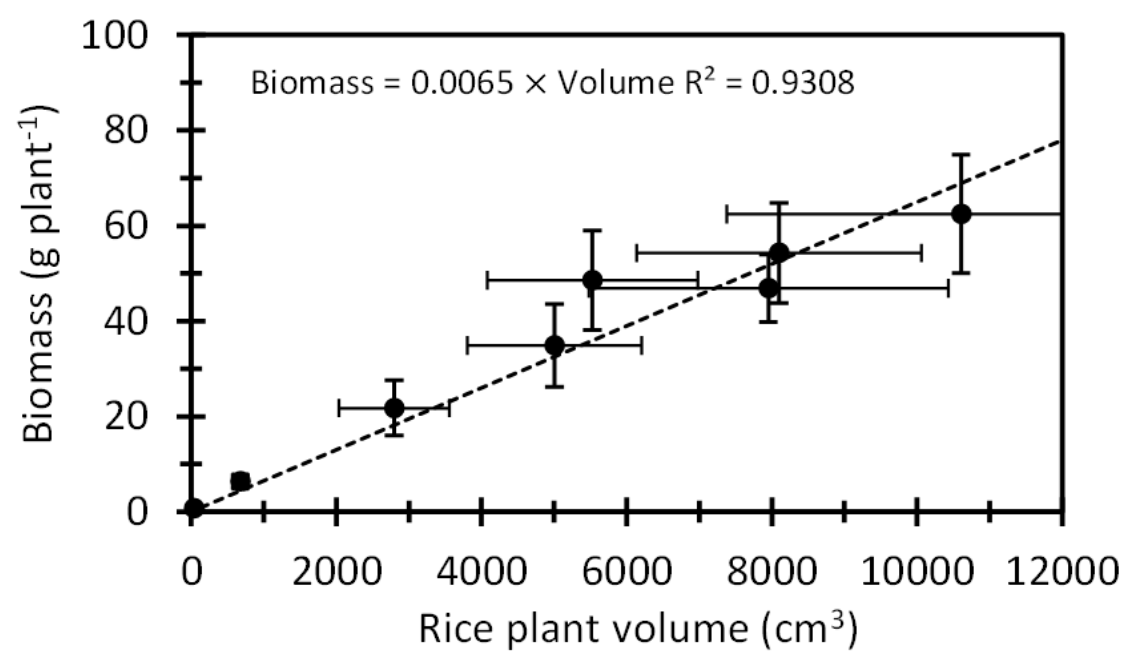

Fig. 5. Relationship between the rice plant volume and the biomass (the above ground biomass without panicles). The data represent the daily average values on the date of rice sampling from the field. Numbers of data to calculate the average and standard deviation (error bars) are ten for the biomass, and four for the rice plant volume, respectively.

The standard deviation of the rice plant volume was large due to the variability of the rice stem diameter in each plant (Fig. 4). To put it more correctly, because the radius is squared to find the cylindrical volume, the effect of difference in the stem diameter was emphasized. It is most likely that traits of panicle (i.e. its weight per volume) is different from the others, and thus the panicle weight was excluded from the analysis. In other words, the biomass hereafter means that the sum of the weight of leaves and stem (the above ground biomass without panicles). The observed high $\mathrm{R}^{2}$ of 0.93 in the linear regression model through the origin indicates that the rice plant volume allow to determine the rice biomass. By applying the linear regression result, the continuous rice biomass were estimated from the daily rice plant volume, which were calculated from the daily rice plant height and stem diameter. The estimated rice biomass showed considerable seasonal variation (Fig. 6). Overall, the biomass increased continuously from around zero to a maximum of ca. $70 \mathrm{~g} \mathrm{plant}^{-1}$ in early August, when both the rice plant height and stem diameter reached to the maximum, and decreased gradually to a value of ca. $35 \mathrm{~g}$ plant $^{-1}$ due to senescence of leaves and outer side of stem. This kind of continuous time series of the rice biomass data cannot be obtained by the direct/destructive plant sampling, in other word the manual plant phenotyping. The proposed method can be considered as the useful tool of the plant phenotyping with advantages such as: nondestructive method to measure the rice plant height, stem diameter and biomass at outdoor, enabling to obtain the continuous time series of the rice plants' data under field conditions, relatively low cost and less laborious work compared with the other methods.

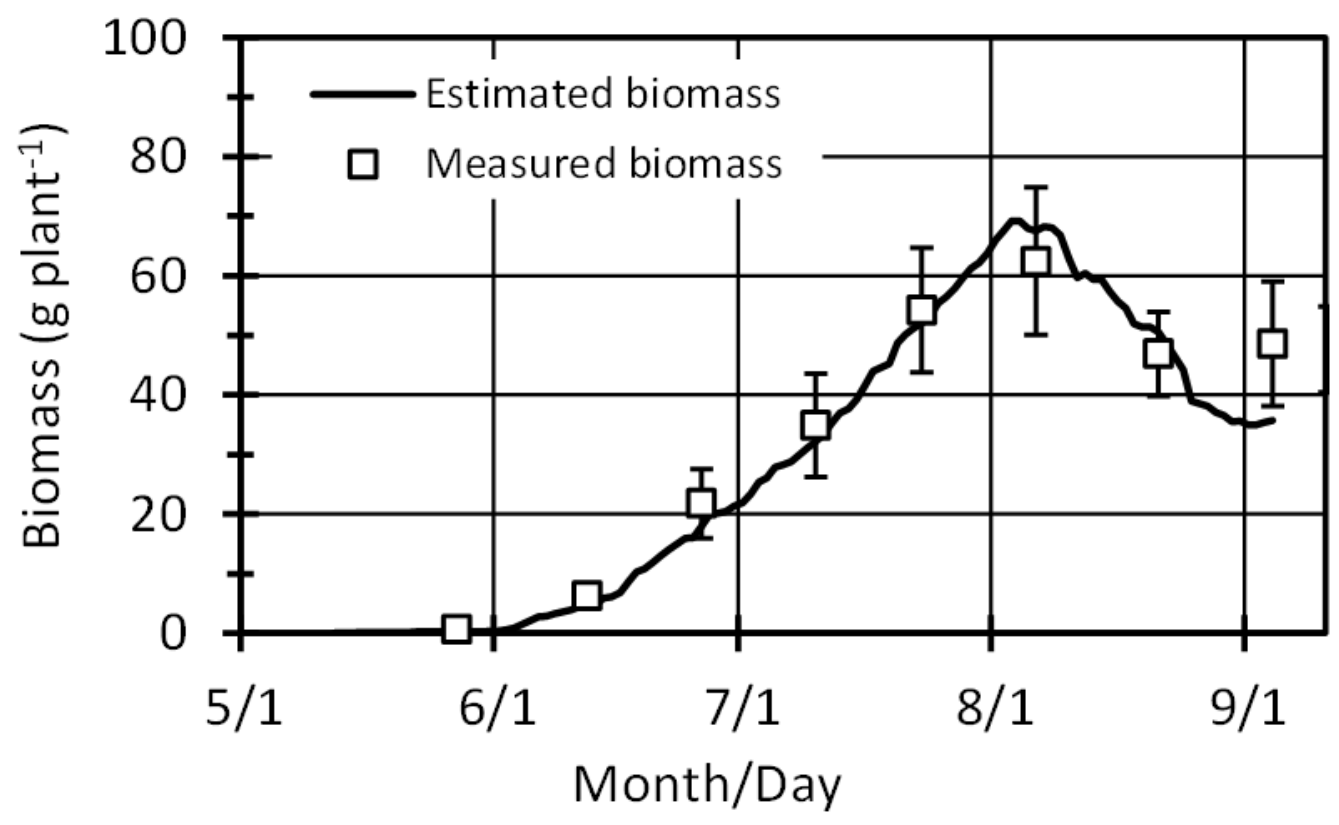

Fig. 6. Seasonal course of the daily biomass (the above ground biomass without panicles) (solid line). The sampled biomass data (square) is presented for reference only 
A point to notice is that the unplanted area between the camera and the target rice plants (Fig. 1) may have caused a phenomenon known as the border effect, also called as the edge effect or the marginal effect, which is the phenomenon that plants in the outermost row next to the unplanted area showed a general increase in yield and growth as compared with the inner row (Gomez and De Datta, 1971; Gomez, 1972). Because dry weights of the target rice plants were not considered this study, the relationship between the rice volume and the biomass of the target rice plants are unknown. Yet, the result in Fig. 6 indicated that the degree of the border effect to the rice volume should be proportional to the biomass because the two variables in Fig. 6 showed the good linear correlation. A further investigation with respect to the border effect will be to provide a proper relation of the rice plant volume to the rice biomass that produce more accurate rice biomass estimation.

\section{Conclusions}

To estimate the continuous data of the plant height, the stem diameter and the biomass of the rice plant, the phenotyping method using the digital time-lapse camera was developed. The method suitably evaluated the rice plant height and stem diameter from the camera images with the small difference to the directly measured ones (data pairs were within one standard deviation). Assuming the shape of rice plant is cylindrical, the rice plant volume was calculated from the height and stem diameter. The relationship between the rice plant volume and the above ground biomass without panicles that were determined from the ten samples from the field indicated that the linear correlation with the high $\mathrm{R}^{2}$ of 0.93 . By using this relationship, the seasonal course of the rice plant biomass can be obtained. Overall, the developed method has several advantages such as nondestructive under field condition, evaluable the several plant traits (i.e. height, stem diameter and biomass), enabling continuous measurement, and relatively low cost and less laborious, and should be one of the useful tools for the plant phenotyping.

\section{Acknowledgements}

The authors thank Mr. Fumio Sasaki for the permission to use his rice field.

\section{References}

Araus, J. L. and Cairns, J. E. 2014. Field high-throughput phenotyping: the new crop breeding frontier. Trends in Plant Science, 19: 52-61.

Constantino, K. P.; Gonzales, E. J.; Lazaro, L. M.; Serrano, E. C. and Samson, B. P. 2015. Plant height measurement and tiller segmentation of rice crops using image processing. Proceedings of the DLSU Research Congress, 3: 1-6.

Dhondt, S.; Wuyts, N. and Inze, D. 2013. Cell to whole-plant phenotyping: the best is yet to come. Trends Plant Science. 18: 428-439.

Friedli, M.; Kirchgessner, N.; Grieder, C.; Liebisch, F.; Mannale, M. and Walter, A. 2016. Terrestrial 3D laser scanning to track the increase in canopy height of both monocot and dicot crop species under field conditions. Plant Methods, 12, 9, DOI 10.1186/s13007-016-0109-7.

Furbank, R. T. and Tester, M. 2011. Phenomics technologies to relieve the phenotyping bottleneck. Trends Plant Science, 16: 635-644.

Gomez, K. A. 1972. Border effects in rice experimental plots. II. Varietal competition. Experimental Agriculture, 8: 295-298.

Gomez, K. A. and De Datta, S. K. 1971. Border effects in rice experimental plots. I. Unplanted borders. Experimental Agriculture, 7: 87-92.

Hartmann, A.; Czauderna, T.; Hoffmann, R.; Stein, N.; and Schreiber, F. 2011. HTPheno: An image analysis pipeline for high-throughput plant phenotyping. BMC Bioinformatics, 12: 148 doi:10.1186/1471-2105-12-148.

Jiang, N.; Yang, W.; Duan, L.; Chen, G.; Fang, W.; Xiong, L. and Liu, Q. 2015: A nondestructive method for estimating the total green leaf area of individual rice plants using multi-angle color images. Journal of Innovative Optical Health Sciences, 8(2): 1550002-1-12.

Li, L.; Zhang, Q.; and Huang, D. 2014. A review of imaging techniques for plant phenotyping. Sensors, 14: 20078-20111.

Mano, M.; Miyata, A.; Yasuda, Y.; Nagai, H.; Yamada, T.; Ono, K.; Saito, M. and Kobayashi, Y. 2007. Quality Control for the Open-path Eddy Covariance Data. Journal of Agricultural Meteorology, 63: 125-138.

Mano, M. 2017. Precise and Continuous Measurement of Plant Heights in an Agricultural Field using a Time-Lapse Camera. Journal of Agricultural Meteorology, 73(3), in press.

McCouch, S.; Baute, G. J.; Bradeen, J.; Bramel, P.; Bretting, P. K.; Buckler, E.; Burke, J. M.; Charest, D.; Cloutier, S.; Cole, G. and et al., 2013. Agriculture: feeding the future. Nature, 499: 23-24.

Minervini, M.; Scharr, H. and Tsaftaris, S. A. 2015. Image analysis: The new bottleneck in plant phenotyping. IEEE Signal Processing Magazine, 32(4): 126-131, doi:10.1109/MSP.2015.2405111.

Miyata, A.; Iwata, T.; Nagai, H.; Yamada, T.; Yoshikoshi, H.; Mano, M.; Ono, K.; Han, G. H.; Harazono, Y.; Ohtaki, E.; Baten, M. A.; Inohara, S.; Takimoto, T.; and Saito, M. 2005. Seasonal variation of carbon dioxide and methane fluxes at single cropping paddy fields in central and western Japan. Phyton, 45: 89-97.

Ono, K.; Miyata, A. and Yamada, T. 2008. Apparent downward $\mathrm{CO}_{2}$ flux observed with open-path eddy covariance over a non-vegetated surface. Theoretical and Applied Climatology, 92: 195-208.

Ono, K.; Miyata, A. and Mano, M. 2013: Mase flux measurement site. Agricultural Meteorology in Kanto, 39: 10-12.

Ono, K.; Mano, M.; Han, G. H.; Nagai, H.; Yamada, T.; Kobayashi, Y.; Miyata, A.; Inoue, Y. and Lal, R. 2015. Environmental Controls on Fallow Carbon 
Dioxide Flux in a Single-Crop Rice Paddy, Japan. Land Degradation \& Development, 26: 331-339.

Saito, M.; Miyata, A.; Nagai, H. and Yamada, T. 2005. Seasonal variation of carbon dioxide exchange in rice paddy field in Japan. Agricultural and Forest Meteorology, 135: 93-109.

Schima, R.; Mollenhauer, H.; Grenzdörffer, G.; Merbach, I.; Lausch, A.; Dietrich, P. and Bumberger, J. 2016. Imagine all the plants: evaluation of a light-field camera for on-site crop growth monitoring. Remote Sensing, 8: 823, doi:10.3390/rs8100823.

Shinozaki, K.; Yoda, K.; Hozumi, K. and Kira, T. 1964a. A quantitative analysis of plant form - the pipe model theory I. Basic analysis. Japanese Journal of Ecology, 14(3): 97-105.

Shinozaki, K.; Yoda, K.; Hozumi, K. and Kira, T. 1964b. A quantitative analysis of plant form - the pipe model theory II. Further evidence of the theory and
ISSN 1999-7361

its application in forest ecology. Japanese Journal of Ecology, 14(4): 133-139.

Sritarapipat, T.; Rakwatin, P. and Kasetkasem, T. 2014. Automatic rice crop height measurement using a field server and digital image processing. Sensors, 14: 900-926, doi:10.3390/s140100900.

Tilly, N.; Hoffmeister, D.; Cao, Q.; Huang, S.; Lenz-Wiedemann, V.; Miao, Y. and Bareth, G. 2014. Multitemporal crop surface models: accurate plant height measurement and biomass estimation with terrestrial laser scanning in paddy rice. Journal of Applied Remote Sensing, 8: 083671-1-22.

Yang, W.; Duan, L.; Chen, G.; Xiong, L. and Liu, Q. 2013. Plant phenomics and high-throughput phenotyping: accelerating rice functional genomics using multidisciplinary technologies. Current Opinion in Plant Biology, 16(2): 180-187. 